\title{
„Sie wissen ja, welche Schätze sie im Kollegium haben“" - Der Einsatz förderpädagogischer Lehrkräfte in Grundschulen aus der Perspektive der Educational-Governance
}

\author{
Lisa Marie Wolf $(\mathbb{D} \cdot$ Torsten Dietze (D) Vera Moser $(\mathbb{D}) \cdot$ Jan Kuhl $(\mathbb{D}$
}

Eingegangen: 1. März 2021 / Angenommen: 18. August 2021 / Online publiziert: 18. Januar 2022

(C) Der/die Autor(en) 2022

Zusammenfassung Der Beitrag untersucht aus der Perspektive der Educational Governance, wie und durch welche Akteure des Mehrebenensystems Schule der Einsatz förderpädagogischer Lehrkräfte an Grundschulen im Kontext inklusiver Bildung gesteuert wird. Die Grundlage hierfür bilden Erhebungen in vier deutschen Bundesländern, die von 2018 bis 2021 durchgeführt wurden. Es zeigt sich eine als „fragmentarisch“ bezeichnete Steuerung, die sich vornehmlich auf Maßnahmen der Inputsteuerung beschränkt und Elemente der Outputsteuerung weitgehend außen vor lässt. Innerschulisch können verschiedene Verhandlungsmodi über den Einsatz der förderpädagogischen Lehrkräfte gefunden werden. Es erfolgt eine Verlagerung der Verantwortung für die Ausgestaltung inklusiver Bildung auf die Einzelschule und insbesondere auf die förderpädagogischen Lehrkräfte.

Schlüsselwörter Educational Governance · Inklusion · Schule ·

Mehrebenensystem · Inklusive Bildung · Steuerung

Lisa Marie Wolf, M.A. ( $₫) \cdot$ Prof. Dr. Jan Kuhl

Fakultät Rehabilitationswissenschaften, Unterrichtsentwicklungsforschung mit dem Schwerpunkt Inklusion, Technische Universität Dortmund, Dortmund, Deutschland

E-Mail: lisamarie.wolf@tu-dortmund.de

Dr. Torsten Dietze · Prof. Dr. Vera Moser

Institut für Sonderpädagogik, Kathrin und Stefan Quandt Stiftungsprofessur für Inklusionsforschung, Goethe-Universität Frankfurt am Main, Frankfurt am Main, Deutschland

E-Mail: dietze@em.uni-frankfurt.de 


\title{
"They know what diamonds they have in staff"-The job clarification of special educational needs teachers in primary schools from an educational governance perspective
}

\begin{abstract}
From the perspective of educational governance, the paper examines how and by which actors of the multilevel school system the job clarification of special educational needs teachers in primary schools in the context of inclusive education is regulated. The analysis is based on surveys conducted in four german federal states from 2018 to 2021. The results show a "fragmentary form" of management, which is primarily limited to measures of input control and largely excludes elements of output control. Within the schools, various modes of negotiation about the use of special educational needs teachers were found. The responsibility for the design of inclusive education is shifted to the individual school and in particular to the special educational needs teachers.
\end{abstract}

Keywords Educational governance - Inclusion - School - Multi-level system · Inclusive education $\cdot$ Management

\section{Einleitung}

Mit der Ratifikation der Behindertenrechtskonvention der Vereinten Nationen (UNBRK) im Jahr 2009 verpflichtet sich Deutschland, ein inklusives Bildungssystem zu gewährleisten, in dem Schülerinnen und Schüler mit und ohne sonderpädagogischen Unterstützungsbedarf gemeinsam unterrichtet werden. Seitdem ist eine deutliche Abkehr von dem jahrzehntelang auf Separation angelegten System sonderpädagogischer Förderung und eine Erhöhung der Inklusionsquoten festzustellen. Gleichzeitig werden auch die förderpädagogischen Lehrkräfte (im Folgenden abgekürzt als FLK) vermehrt in Regelschulen eingesetzt: In Nordrhein-Westfalen (NW) waren im Schuljahr 2008 erst 12\% der FLK (statistisch, Zählung nach Lehrämtern und nicht Einzelpersonen) an einer Regelschule tätig, 2018 waren es bereits 33\% (MSW NRW 2009, S. 48; MSB NRW 2019, S. 56).

Die Modellversuche der 1980er-Jahre und die erste, gesetzlich verankerte Umsetzung von Integration ab den 1990er-Jahren gingen mit einer vergleichsweisen großzügigen Ausstattung mit FLK einher und inklusiver Unterricht wurde insbesondere als Unterricht in Doppelbesetzung realisiert. Hingegen steht die heutige inklusive Schulentwicklung unter dem Druck eines Fachkräftemangels. So gelang es bspw. in Niedersachsen im Jahr 2016 die förderpädagogische Unterrichtsversorgung nur zu etwa $62 \%$ mit sonderpädagogischer Expertise zu hinterlegen (LRH NI 2018, S. 47). Die professionelle Rolle der FLK in Regelschulen wird unter anderem auch deswegen nicht mehr (allein) in den einzelnen Teams der Doppelsteckung verhandelt, sondern erfordert eine gezielte Einbettung in die Organisation Schule (Grummt 2019) sowie eine passgenaue Professionalisierung der beteiligten Akteure. In Befragungen der FLK zu ihrem Einsatz an Regelschulen zeigen sich spezifische, vor allem förder- und beratungsbezogene, jedoch insgesamt heterogene Aufgabenbereiche (vgl. Neumann 2019, S. 69). 
In diesem Beitrag wird untersucht, von wem und inwieweit der Einsatz von FLK im Rahmen inklusiver Bildung an Regelschulen gesteuert wird. Grundlage der Untersuchung bieten Daten aus dem Projekt Förderpädagogische Lehrkräfte in inklusiven Schulen (FoLiS), in dem von 2018 bis 2021 in einem Mixed-MethodsDesign Vertreterinnen und Vertreter der Ebenen des Schulsystems (Bildungsministerien, Staatliche Schulämter, Grundschulleitungen, FLK) in vier Bundesländern befragt wurden. Auf der Mesoebene wurden hierbei explizit Grundschulen, auf der Mikroebene die Perspektive der FLK (nicht der Grundschullehrkräfte) fokussiert. Theoretische Grundlage der Analyse bildet ein Mehrebenenmodell des Schulsystems sowie die Perspektive der Educational Governance (im Folgenden abgekürzt als EG, Kap. 2). Anhand von Daten aus dem Forschungsprojekt FoLiS (Kap. 3) wird exemplarisch beantwortet, wie die zuvor analysierten Steuerungsformen der Makro- und Exoebene (Schulpolitik und Bildungsverwaltung) und die sich daraus ergebenden Kontexte auf der Meso- und Mikroebene bearbeitet werden (Kap. 4).

\section{Steuerung und Educational Governance}

\subsection{Steuerung und schulische Inklusion}

Unter Steuerung wird die „Praxis intentionalen (steuernden) Handelns“ verstanden (Gasterstädt 2019, S. 12), mit der Strukturen im Mehrebenensystem Schule bewahrt, verändert oder gelenkt werden sollen (Mayntz 1997, S. 191). Steuerung bedeutet dabei, dem inzwischen vorherrschenden ,Steuerungsskeptizismus“ (Berkemeyer 2010, S. 90) entsprechend, nicht, dass „Steuerungsintentionen zu $100 \%$ in entsprechende Folgehandlungen umgesetzt würden“, sondern umfasst auch die Berücksichtigung von Nebenwirkungen des Steuerungshandelns, transintentionalen Effekten sowie individuellen und sozialen Vermittlungsschritten (Altrichter und Maag-Merki 2016, S. 6).

Im Sinne eines Abschieds von ,plandeterminierten Vorstellungen von Steuerung“ (ebd.) definieren Altrichter und Maag-Merki (2016) Eckpunkte eines erweiterten Steuerungsverständnisses, in denen Besonderheiten und Herausforderungen von Steuerungsvorhaben im Mehrebenensystem Schule sichtbar werden: Zum einen beteiligen sich viele Akteure auf verschiedenen Hierarchieebenen mit verschiedenen Intentionen und unterschiedlicher Gestaltungsmacht an der Entwicklung des Systems. Steuerung ist demzufolge als die Summe der ,Transaktionen aller relevanten Systemmitspieler*innen“ zu verstehen (ebd., S. 6). Zum anderen entwickeln sich die Zielsysteme der Steuerungsbemühungen und die beteiligten Akteure auch ohne systematisches Eingreifen nach einer eigenen Logik. Sie halten nicht „still“, sondern nehmen politische, rechtliche oder gesellschaftliche Anforderungen selektiv und aktiv-konstruktiv auf und übersetzen sie in die jeweiligen Systempraktiken (ebd., S. 4f.). Dabei versuchen viele Steuerungsakteure Entwicklungen im Sinne ihrer Intentionen rational zu steuern, so dass nicht alle Informationen reflexiv erfasst und einbezogen werden können. Auch aufgrund der Überlagerung von Steuerungsprozessen sind ,,viele bedeutsame Dynamiken und Wirkungen ihrer Handlungen transintentional“" (ebd., S. 6) und können - auf derselben oder auf anderen Ebenen - 
unintendierte Konsequenzen nach sich ziehen und neue Voraussetzungen schaffen (vgl. das Konzept der Rekontextualisierung von Fend 2008). Für Reformprozesse im Mehrebenensystem Schule bedeutet dies, dass sie nicht direkt „hineingesteuert“ werden können (Altrichter und Maag-Merki 2016, S. 6). Steuerung soll diese autonome Dynamik eines Systems vielmehr gezielt (um)lenken, also etwa Strukturen bewahren oder bestehende Strukturen verändern (Mayntz 1997, S. 191).

Unter den Leitideen des New Public Managements (NPM) - ein Konzept, das auf ein unterstelltes „Leistungsmanko der staatlichen Bürokratie“ reagiert (Kussau und Brüsemeister 2007, S. 20) - wurde der Annahme einer begrenzten Steuerbarkeit im Schulsystem Rechnung getragen, so dass in Deutschland seit etwa 25 Jahren diverse Maßnahmen einer veränderten Systemsteuerung unter dem Begriff Governance diskutiert und eingeführt werden (Fuchs 2008, S. 19). Kern dieser Veränderungsprozesse ist der systematische Übergang von einer input- zu einer outputorientierten Steuerung (Standards-Based-Reform). Der Begriff des Outputs wird im schulischen Kontext ,in Zusammenhang mit der Steuerung des Bildungssystems mittels Standards, Vergleichsarbeiten und Evaluationen verwendet" (KMK For.mat o.J.). Schulische Inklusion findet jedoch bisher nur teilweise und unsystematisch Berücksichtigung in den entwickelten Instrumenten der Outputsteuerung (z. B. in Bildungsstandards, standardisierten Schulevaluationen, Holder und Kessels 2018; Schulinspektionen, Piezunka 2020, S. 224). Ihre normative Bestimmung steht vor dem Dilemma ,einerseits theoretisch idealistisch $\mathrm{zu}$ argumentieren und andererseits oftmals schon in der Definition dessen, was Inklusion ausmachen soll, pragmatische Abstriche zu machen, um sich nicht der Realitätsferne bezichtigen zu lassen“ (Heinrich et al. 2013, S. 73 f.). So findet die Umsetzung der schulischen Inklusion im Kontext der Entwicklung und Steuerung des allgemeinen Schulsystems statt, welches weiterhin an seinen auf Leistung und Leistungsfeststellung beruhenden, separierenden Funktionen festhält, die nur bedingt mit der Umsetzung eines inklusiven Schulsystems vereinbar sind (Budde 2018, S. 49). Dementsprechend folgt Inklusion als Norm auch über zehn Jahre nach Ratifizierung der UN-BRK ,keineswegs einem einheitlichen Verständnis“ (Tegge 2020, S. 32), sondern impliziert vielmehr ,diverse, nicht immer deckungsgleiche Anforderungen an ein Schulsystem“ (ebd.). Ergebnis sind nicht nur international, sondern auch national unterschiedliche Konzepte von Inklusion und daran anschließend unterschiedliche Gesetzgebungen (Gasterstädt 2019, S. 3).

Folgende Annahme lässt sich hieraus ableiten:

Im Kontext der Leitideen des NPM ist die Inputsteuerung auch für das Reformanliegen Inklusive Bildung - bei gleichzeitig bestehender Schulautonomie (Rürup 2020) - im Wesentlichen auf eine grundlegende Schulstruktur, die Ausbildung der Lehrkräfte, Bildungsgänge, Lehrpläne und Stellenzuweisungen beschränkt. Im Zuge der Reform hin zu einer outputorientierten Steuerung werden entsprechende Instrumente (z. B. Bildungsstandards, Evaluationen) bereitgestellt in denen jedoch inklusive Schulentwicklung keine umfassende Berücksichtigung findet. Ferner existieren bislang für inklusive Bildung keine spezifischen implementierten Standards oder Konzepte der Qualitätssicherung. Inklusive Bildung findet insofern ohne die Bereitstellung einer Definition und eines 
entsprechenden „Handwerkszeugs“ in einem Schulsystem statt, welches ihren grundlegenden Intentionen widerspricht.

Aus dieser Annahme entsteht die Frage, welche Steuerungskontexte, -möglichkeiten und -ergebnisse bezüglich der Umsetzung der UN-BRK im Mehrebenensystem Schule gefunden werden können und wie diese von den Akteuren der jeweiligen Ebenen angenommen und umgesetzt bzw. weiterbearbeitet werden. Hierfür werden im Folgenden die analytischen Grundlagen beschrieben.

\subsection{Das Mehrebenensystem Schule und Educational-Governance als Analyseperspektive}

Für Analysen von Steuerungsprozessen im Schulsystem sind Ansätze notwendig, die der Komplexität des Systems Rechnung tragen und sowohl die Akteure auf den verschiedenen Ebenen mit ihren jeweiligen Handlungsintentionen, -kontexten und -möglichkeiten als auch Interdependenzen und intendierte oder nicht intendierte Wirkungen einbeziehen. Die Grundlage für derartige Analysen bilden Mehrebenenmodelle des Schulsystems.

Modelle des Mehrebenensystems Schule umfassen in der Regel mindestens eine Makro- (gesellschaftlicher Kontext, Bildungspolitik, Bildungsverwaltung), eine Meso- (Einzelschule) und eine Mikroebene (Lehrkräfte $\left.{ }^{1}\right)$, sind jedoch in ihrer Ausgestaltung vielfältig (z.B. 7-Ebenen-Modell, Berkemeyer 2010). Das hier zu Grunde liegende Vier-Ebenen-Modell von Zlatkin-Troitschanskaia (2006) stellt den Bezug zu Steuerungsprozessen her. Hier wird die Makroebene als „,(regionales) strukturelles (Hervorhebung im Original) Steuerungsumfeld“ verstanden sowie eine Exoebene eingefügt, die explizit das schuladministrative Steuerungshandeln der Schulaufsichten beinhaltet. Die Mesoebene umfasst das Steuerungshandeln auf Einzelschulebene, die Mikroebene bezieht sich auf das pädagogisch professionelle Handeln der Lehrkräfte (Zlatkin-Troitschanskaia 2006, S. 206; siehe Abb. 1).

Abb. 1 Vierebenenmodell des Schulsystems. (Eigene Darstellung in Anlehnung an ZlatkinTroitschanskaia 2006, S. 206)

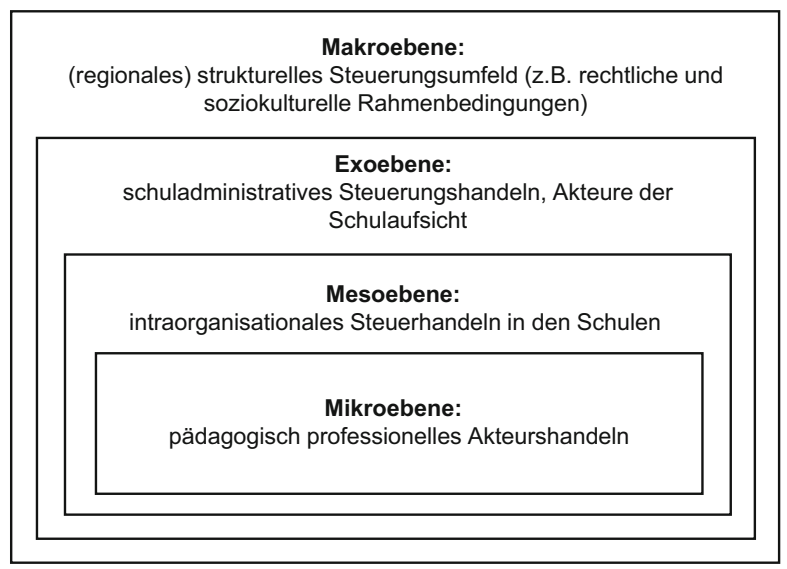

\footnotetext{
1 Eine mögliche „Mikroebene Lernende“ (z. B. bei Tegge 2020, S. 42) wird in diesem Beitrag nicht untersucht.
} 
Eine Möglichkeit, sich Steuerungsprozessen im Mehrebenensystem Schule analytisch zu nähern, ist die Perspektive der EG. Sie bietet einen Analyseansatz, der die Komplexität der Interaktionen und Prozesse zwischen den Ebenen des Schulsystems, ihre Zusammenhangsgefüge und Wirkmechanismen berücksichtigt und über Annahmen einer Top-Down-Steuerung hinausgeht (Gasterstädt 2019, S. 103). Mit ihrer Hilfe können komplexe Fragen der Steuerung im Mehrebenensystem Schule aus einer Metaposition heraus empirisch und theoretisch analysiert werden (Altrichter und Maag-Merki 2016, S. 7 f.; Kussau und Brüsemeister 2007, S. 26).

Sowohl Forschungen zum Konzept der EG als auch die Praxis schulischer Inklusion profitieren von einer Anwendung der EG-Perspektive auf das spezifische Reformanliegen Inklusive Bildung. Zum einen stellt das Reformanliegen, ein inklusives Schulsystem zu entwickeln für die EG-Forschung ein attraktives und ergiebiges Untersuchungsfeld dar, ,um Strategien und Dynamiken der Bildungsreform unter den Bedingungen einer begrenzten Steuerungsfähigkeit der Bildungspolitik und einer begrenzten Steuerbarkeit des Bildungswesens zu analysieren“ (Rürup 2015, S. 684). Zum anderen verspricht die EG-Perspektive ,praktisch relevante Erkenntnisse über die Gelingensbedingungen Inklusiver Bildung als einem anspruchsvollen Reformprogramm im Schulwesen“ (ebd., S. 687). Rürup (2015) konstatiert, dass dennoch bezüglich schulischer Inklusion bisher wenig Forschung aus der EG-Perspektive vorliegt, was er unter anderem darauf zurückführt, dass Inklusion vorrangig als ein ,eher schul- und unterrichtsbezogenes (pädagogisches) Reformanliegen“ verstanden wird, während sich der Governance-Begriff insbesondere auf politischadministrative Steuerungsinstrumente und Koordinationspraxen bezieht, die ,programmatisch kaum tangiert" werden (ebd., S. 687). Es ist daher Anliegen des vorliegenden Beitrags, die Steuerungskontexte, -formen und -ergebnisse der Akteure im Mehrebenensystem Schule aus einer EG-Perspektive in Bezug auf den Einsatz von FLK an Grundschulen zu analysieren.

\subsection{Steuerungskontexte, -formen und -ergebnisse im Mehrebenensystem Schule}

In Anlehnung an das Handlungsmodell von Fend (2008, S. 36f.) lassen sich aus der EG-Perspektive die Kontexte der Handlungen, Handlungsmöglichkeiten und Handlungsergebnisse der Akteure auf den jeweiligen Ebenen des Schulsystems beschreiben. Es bietet eine Grundlage, anhand derer Fragestellungen bzw. Analysen strukturiert und eingeordnet werden können (siehe Tab. 1). Interdependenzen zwischen den Ebenen werden dabei berücksichtigt: ,Auf jeder Handlungsebene [...] sind die Vorgaben auf der jeweils übergeordneten Ebene Umwelten des Handelns, die eine Umsetzung an die jeweils neuen, ebenenspezifischen Besonderheiten der Umwelten des Handelns erfordern“ (Fend 2008, S. 34). „Handlungen“ werden im Sinne dieses Beitrags als „Steuerungshandlungen“ bzw. Steuerung verstanden - im Folgenden werden daher die Begriffe „Steuerungskontexte, -formen und -ergebnisse“ verwendet. Die Steuerungsergebnisse höherer Ebenen strukturieren somit mehr oder weniger, intendiert oder nicht-intendiert den Steuerungskontext der nachfolgenden Ebenen. 
Tab. 1 Handlungs-/Steuerungskontexte, -formen und -ergebnisse im Mehrebenensystem Schule. (Eigene Darstellung in Anlehnung an Fend 2008, S. 36f.)

\begin{tabular}{|c|c|c|}
\hline Ebene & Handlungs-/Steuerungskontext & Handlungs-/Steuerungsformen und -ergebnisse \\
\hline $\begin{array}{l}\text { Makroebene } \\
\text { (Bildungs- } \\
\text { politik) }\end{array}$ & $\begin{array}{l}\text { aftliche Kontexte, kulturelle } \\
\text { n }\end{array}$ & $\begin{array}{l}\text { Gesetze/Verordnungen, Personal- und Sachver- } \\
\text { sorgung }\end{array}$ \\
\hline $\begin{array}{l}\text { Exoebene } \\
\text { (Bildungs- } \\
\text { verwaltung) }\end{array}$ & $\begin{array}{l}\text { Gesetzliche Vorgaben, Erfordernisse } \\
\text { der Schulversorgung, Entscheidungs- } \\
\text { befugnisse }\end{array}$ & $\begin{array}{l}\text { Gesetze/Verordnungen, Ausführungsrege- } \\
\text { lungen, Zuweisung personeller/finanzieller } \\
\text { Ressourcen, Programmentwicklung, Organisa- } \\
\text { tionsmodelle }\end{array}$ \\
\hline $\begin{array}{l}\text { Mesoebene } \\
\text { (Einzelschule) }\end{array}$ & $\begin{array}{l}\text { Organisationsmodelle, Ressourcen, } \\
\text { Schülerschaft, Kollegium }\end{array}$ & $\begin{array}{l}\text { Gestaltungsformen von Schule, schulinterne } \\
\text { Regelungen, Makroorganisation des Unter- } \\
\text { richts, Stundenpläne, Deputatsverteilungen, } \\
\text { Gremien, Schulkultur, Qualitätssicherung }\end{array}$ \\
\hline $\begin{array}{l}\text { Mikroebene } \\
\text { (Lehrkräfte) }\end{array}$ & $\begin{array}{l}\text { Rechtliche Vorgaben (Lehrpläne, } \\
\text { Prüfungsanforderungen), schuli- } \\
\text { sche Vereinbarungen, Merkmale der } \\
\text { Schulklasse, eigene Belastbarkeit }\end{array}$ & $\begin{array}{l}\text { Unterrichtsvorbereitung, Unterricht(en), Klas- } \\
\text { senführung, Erziehung, Konfliktlösen, Bewer- } \\
\text { ten und Beurteilen, durchgeführte Lektionen, } \\
\text { Klassenklima }\end{array}$ \\
\hline
\end{tabular}

\section{Fragestellung und Methodik}

In diesem Beitrag werden auf Grundlage der Daten des Projektes FoLiS Steuerungskontexte, -formen und -ergebnisse auf den verschiedenen Ebenen des Schulsystems bezüglich des Einsatzes von FLK an inklusiven Grundschulen in den Blick genommen. ${ }^{2}$ Aus der in Kap. 2 vorgestellten Annahme lässt sich die Hypothese ableiten, dass die konkreten Praktiken des Einsatzes der FLK an den Grundschulen durch die Akteure der Makro- und Exoebene nur unzureichend gesteuert werden und somit Einzelschulen sowie einzelne Lehrkräfte für die Ausgestaltung des (eigenen) Einsatzes verantwortlich sind. Konkret werden diesbezüglich folgende Fragen bearbeitet:

1. Welche Steuerungsformen finden sich auf der Makro- und Exoebene? (Abschnitt 4.1)

2. Welche Steuerungskontexte ergeben sich hieraus für die Meso- und Mikroebene (Abschnitt 4.2)?

3. Mit welchen Steuerungsformen reagieren die Akteure der:
a. Mesoebene (Abschnitt 4.3) und
b. Mikroebene (Abschnitt 4.4)?

Diese Fragestellungen stellen einen Teil des explorativen Projektes FoLiS dar, in dem von 2018 bis 2021 in einem Mixed-Methods-Design Daten von Schulverwaltungen (qualitativ), Grundschulleitungen (quantitativ) und FLK (quantitativ und qualitativ) aus den vier Bundesländern Hessen (HE), Nordrhein-Westfalen (NW), Berlin (BE) und Mecklenburg-Vorpommern (MV) erhoben wurden (vgl. Tab. 2). Die Auswahl der Bundesländer, die sich unter anderem in der Bevölkerungs- und

\footnotetext{
2 An allen befragten Schulen wurde zum Befragungszeitraum mind. eine Schülerin bzw. ein Schüler mit sonderpädagogischem Förderbedarf beschult.
} 
Tab. 2 Methoden und Stichproben des Forschungsprojektes FoLiS

\begin{tabular}{|c|c|c|c|c|c|c|}
\hline Projektschritt & Methode \& Ebene & Gesamt & NW & $\mathrm{HE}$ & $\mathrm{BE}$ & MV \\
\hline & Leitfadengestützte Experteninterviews: & & & & & \\
\hline $1 \mathrm{a}$ & Bildungsministerien, & 4 & 1 & 1 & $1^{\mathrm{c}}$ & 1 \\
\hline $1 b$ & Bezirksregierungen $^{\mathrm{a}}$ und & 2 & 2 & 0 & 0 & 0 \\
\hline $1 \mathrm{c}$ & Staatliche Schulämter ${ }^{\mathrm{b}}$ & 16 & 4 & 4 & 4 & 4 \\
\hline 2 & $\begin{array}{l}\text { Online-Fragebogen } \\
\text { Grundschulleitungen }\end{array}$ & 89 & 28 & 19 & 20 & 22 \\
\hline $3 a$ & $\begin{array}{l}\text { Paper-Pencil-Fragebogen } \\
\text { förderpädagogische Lehrkräfte }\end{array}$ & 47 & 47 & 15 & 11 & 10 \\
\hline $3 b$ & $\begin{array}{l}\text { Leitfadengestützte Experteninterviews } \\
\text { förderpädagogische Lehrkräfte }\end{array}$ & 40 & 12 & 11 & 10 & 7 \\
\hline
\end{tabular}

aDie Bezirksregierungen in NW sind als obere Schulaufsichtsbehörde den Schulämtern übergeordnet

${ }^{b}$ Auswahl nach Teilnahmebereitschaft: NW: von 53, HE: von 15, BE: von 12, Vollbefragung in MV (vier von vier Schulämtern)

${ }^{\mathrm{c}}$ Schriftliche Antwort

Schuldichte, der sonderpädagogischen Förderquote und ihrer Integrationshistorie unterscheiden, erfolgte, um eine möglichst breite Perspektive auf die Möglichkeiten des Einsatzes von FLK an Grundschulen einzunehmen. Die einbezogenen Schulämter wurden ebenfalls mit dem Ziel einer möglichst großen Heterogenität ausgewählt. Die Befragung der Grundschulleitungen sowie nachfolgend der FLK erfolgte jeweils ohne Beschränkung auf diese Schulamtsbereiche. Die befragten FLK arbeiteten an Schulen, in denen zuvor die Grundschulleitungen befragt wurden. Tab. 2 zeigt eine Übersicht über die Erhebungsmethoden und Stichproben in den jeweiligen Projektschritten. Die Erhebungsinstrumente wurden jeweils auf Grundlage der im vorherigen Projektschritt gewonnenen Erkenntnisse entwickelt bzw. angepasst.

Das Erkenntnisinteresse des Gesamtprojektes bezog sich zum einen auf die hier dargestellten Steuerungsformen bezüglich des Einsatzes der FLK auf den Ebenen des Schulsystems, zum anderen auf konkrete Ergebnisse auf der Ebene der individuellen FLK, so dass eine Vielzahl von Erhebungsinstrumenten und Variablen verwendet wurde (z.B. zu Aufgaben und Zuständigkeiten der FLK und Aufgabenverteilungen mit den Grundschullehrkräften, Differenzierungsformen, berufliche Zufriedenheit, Belastungserleben, inklusionsbezogene Beliefs). Tab. 3 zeigt eine Übersicht über die zur Beantwortung der Fragestellungen dieses Beitrags -1 bis $3 b$ (erste Spalte) herangezogenen Daten, die konkret verwendeten Inhalte der Interviews und Fragebögen sowie die jeweiligen Auswertungsformen (Hinweis: eine Beschreibung des methodischen Vorgehens im gesamten Forschungsprojekt kann dem Abschlussbericht entnommen werden, vgl. Kuhl et al. 2021). 
Tab. 3 Zur Beantwortung der Fragestellungen 1 bis $3 \mathrm{~b}$ verwendete Daten, Inhalte und Auswertungsformen aus dem Projekt FoLiS

\begin{tabular}{|c|c|c|c|}
\hline & $\begin{array}{l}\text { Projektschritt/ } \\
\text { verwendete Daten } \\
\text { (vgl. Tab. 2) }\end{array}$ & Verwendete Inhalte/Angaben & Auswertungsform \\
\hline 1 & $\begin{array}{l}\text { 1a, b, c-Schul- } \\
\text { verwaltungen }\end{array}$ & $\begin{array}{l}\text { Aufgaben und Steuerungsformen bezüglich der } \\
\text { Steuerung des Einsatzes der FLK an Grundschulen }\end{array}$ & $\begin{array}{l}\text { Quantitativ inhalts- } \\
\text { analytisch }\end{array}$ \\
\hline \multirow[t]{2}{*}{2} & $\begin{array}{l}2-\text { Grundschul- } \\
\text { leitungen }\end{array}$ & $\begin{array}{l}\text { Einschätzung der und objektive Indikatoren zur } \\
\text { personellen Ausstattung mit FLK, Transparenz } \\
\text { der Zuweisungskriterien, Zufriedenheit mit der } \\
\text { Unterstützung durch die Staatlichen Schulämter }\end{array}$ & $\begin{array}{l}\text { Deskriptiv, } \\
\text { inferenzstatistisch } \\
\text { auf Bundeslandun- } \\
\text { terschiede }\end{array}$ \\
\hline & $\begin{array}{l}3 \mathrm{a}-\text { FLK (Frage- } \\
\text { bögen) }\end{array}$ & Einschätzung der personellen Ausstattung mit FLK & \\
\hline $3 a$ & $\begin{array}{l}2 \text { - Grundschul- } \\
\text { leitungen }\end{array}$ & $\begin{array}{l}\text { Vorliegen fester Besprechungs- und Planungszei- } \\
\text { ten, Teilnahme der FLK an Gesamt- bzw. Lehrer- } \\
\text { und Fachkonferenzen, Nutzung von schriftlichen } \\
\text { Vereinbarungen }\end{array}$ & $\begin{array}{l}\text { Deskriptiv, infe- } \\
\text { renzstatistisch auf } \\
\text { Bundeslandunter- } \\
\text { schiede }\end{array}$ \\
\hline $3 b$ & $\begin{array}{l}3 \mathrm{~b}-\mathrm{FLK}(\text { Inter- } \\
\text { views) }\end{array}$ & $\begin{array}{l}\text { Innerschulische Aushandlungsprozessen über die } \\
\text { Einsatzpraktiken der FLK }\end{array}$ & $\begin{array}{l}\text { Quantitativ inhalts- } \\
\text { analytisch }\end{array}$ \\
\hline
\end{tabular}

\section{Ergebnisse}

\subsection{Steuerung auf der Makro- und Exoebene (Fragestellung 1)}

Aus der Dokumentenanalyse sowie aus den Interviews mit den Expertinnen und Experten aus der Makro- und Exoebene konnten folgende Erkenntnisse gewonnen werden:

Da auf nationaler Ebene als Steuerungsaktivität lediglich Empfehlungen (ohne bindende Wirkung) zu einer qualitativ hochwertigen Lehrkräftebildung als Steuerungsaktivität vorliegen (KMK 2011, 2014; HRK und KMK 2015), liegen alle weiteren Steuerungshandlungen im Sinne der föderalen Bildungshoheit bei den Bundesländern. Auf Ebene der Bundesländer verantworten die Akteure der Makro- und Exoebene die grundlegenden schulischen Inklusionskonzepte, Organisationsmodelle der sonderpädagogischen Förderung inklusive der Errichtung von (neuen) Unterstützungssystemen und konkrete Ressourcenzuweisungsmodelle für Lehrkraftstunden. Diesbezügliche Regelungen sind zunächst in den Landesschulgesetzen festgelegt und des Weiteren in den Inklusionsstrategien der Länder dokumentiert, die fortwährend ergänzt und angepasst werden. Konkret verbunden sind hiermit auch Klärungen über die Schulzugehörigkeit der FLK, einen möglichen Einsatz als Klassenlehrkraft und die Zuständigkeit für sonderpädagogische Diagnostik.

Aus den Interviews konnten folgende allgemeine formale bzw. systematische zentrale Handlungs- und Steuerungsformen der Akteure der Exoebene herausgearbeitet werden (die interviewten Expertinnen und Experten aus den Schulämtern der Bundesländer waren teilweise die Schulamtsleitungen, teilweise Fachreferentinnen bzw. -referenten für Inklusive Bildung und teilweise Referentinnen bzw. Referenten für Grundschulen, hierdurch bedingt unterschieden sich die Blickwinkel der interviewten Personen im Spezifischen): 
- Die Erhebung der Bedarfe an FLK-Stellen (inklusive der benötigten spezifischen sonderpädagogischen Qualifikationen, z.B. Förderschwerpunkt „Hören“ oder „Sprache“) an den Grundschulen zusammen mit den Schulleitungen im Aufsichtsbereich. Dies mündet in schulscharfen Bedarfsanalysen und konkreten Stellenanforderungen.

- Die exakte Erstellung der schulspezifisch maximalen Ressourcenzuweisung für FLK auf Basis der im Schulamtsbereich vorliegenden Kennzahlen. Die Steuerungsvorgaben der Makroebene zur Ressourcenverteilung der FLK werden in Ansprüche auf Stellen(-anteile) übersetzt.

- Die Abfrage an Förderschulen und -zentren sowie anderen Regelschulen, ob und welche FLK für den Einsatz im inklusiven Unterricht einer Grundschule mit Stellenanteilen oder vollständig freigegeben werden können. Um FLK für eher seltene Förderschwerpunkte wie z.B. „Sehen“ zu rekrutieren, erfolgt die Abfrage auch über den eigenen Aufsichtsbereich hinaus.

- Die Übernahme einer Koordinationsfunktion, um FLK und Schulen ,zusammenzubringen“. Die endgültige Auswahl der FLK erfolgt dabei in BE, MV und NW aufgrund einer eingeräumten Autonomie in Personalfragen weitgehend durch die Schulleitungen der Grundschulen. In HE sind auch die Beratungs- und Förderzentren (BFZ) in die Lehrkräftezuweisung involviert.

Es wird erkennbar, dass sich die Steuerungsformen der Akteure der Exoebene bezüglich des Einsatzes der FLK vornehmlich auf die Ressourcenzuweisung im Rahmen ihre jeweiligen Steuerungskontexte (vgl. Tab. 1) beziehen. Eine weitere Steuerung des Einsatzes auf einer Inhaltsebene erfolgt nicht. Als Begründung für die geringe inhaltliche Steuerung wurde in den Interviews unter anderem die Heterogenität der schulischen Einsatzbedingungen benannt, so dass das Schulamt keine detaillierten Vorgaben zu Aufgabengebieten machen könne (Interview L1_C_T3). Weiter wurde auf die Schulautonomie und die (angenommene) Führungskompetenz der Schulleitungen verwiesen:

Ja, das ist auch originäre Aufgabe der Schulleitung. [...] Einsatzplanung für Lehrkräfte. Und sie kennen ja ihre Kolleginnen und Kollegen und wissen auch, welche Schätze sie im Kollegium haben. (Interview L1_C_T4)

In einigen Interviews wurde berichtet, dass die Frage einer Arbeitsplatzbeschreibung häufig an die Schulverwaltung herangetragen wird. Während der Laufzeit des Projektes konnte festgestellt werden, dass zum Teil tatsächlich Aufgabenbeschreibungen durch Akteure der Exoebene veröffentlicht wurden (z. B. Arbeitsstelle Inklusion der Bezirksregierung Köln 2019). Bei einer Durchsicht dieser Veröffentlichungen findet sich jedoch - wie auch in den Interviews mit den Expertinnen und Experten der Exoebene - eine Delegation der Steuerung des Einsatzes der FLK an die Akteure der Mesoebene:

Leitlinie für den Einsatz von sonderpädagogischen Lehrkräften sollte immer die effektive Nutzung der sonderpädagogischen Expertise sein. Aus diesem Grund ist es ggf. gewinnbringend, die Lehrkräfte der Sonderpädagogik inner- 
halb ihrer verpflichtenden Unterrichtsstunden flexibler einzusetzen. Je nach Ressource kann es sinnvoll sein, z. B. Schülergruppen oder Klassen epochal oder zeitlich begrenzt durch die Lehrkraft der Sonderpädagogik zu fördern oder aber flexible Beratungskonzepte zu entwickeln. (Manual der Bezirksregierung Düsseldorf 2020, S. 32)

Eine Konkretisierung der Aufgabenbereiche und Zuständigkeiten der FLK findet sich seit August $2020 \mathrm{im}$,Leitfaden zu den Aufgaben und der Organisation der inklusiven Schulbündnisse“ des Hessischen Kultusministeriums (HKM 2020). Insgesamt kann für den Erhebungszeitraum des Projekts FoLiS (2018-2021) jedoch festgestellt werden, dass Steuerungsformen auf der Makro- und Exoebene bezüglich des Einsatzes der FLK vornehmlich die grundlegende Einsatzstruktur und das Verfahren der Ressourcenzuweisung umfassen. Handlungsempfehlungen (keine Standards!) werden allmählich und vermutlich vor allem auf Drängen der Akteure der Meso- und Mikroebene bereitgestellt, eine weitere Definition möglicher Outputs schulischer Inklusion (bspw. Standards) erfolgt nicht. Die Delegation der Verantwortung für die Ausgestaltung des Einsatzes der FLK erhält in den erstellten Handlungsempfehlungen teilweise (bspw. im oben erwähnten Manual der Bezirksregierung Düsseldorf) einen ,offiziellen Charakter".

Den Grundschulleitungen wurden jedoch in allen vier untersuchten Bundesländern inzwischen mit speziellen Aufgaben betraute Akteure für die Entwicklung der inklusiven sonderpädagogischen Förderung und explizit als Ansprechpartner bereitgestellt (im Projekt nicht befragt). Diese neuen Akteure lassen sich nicht immer eindeutig der Exo- oder Mesoebene zuordnen (z. B. NW: Inklusionskoordinatorinnen bzw. -koordinatoren im Schulamt ohne schulaufsichtliche Funktion, BE: Schulpsychologische und Inklusionspädagogische Beratungs- und Unterstützungszentren (SIBUZ)). Ihre Aufgaben sind in den Inklusionsstrategien der Länder hinterlegt.

\subsection{Steuerungskontexte für die Meso- und Mikroebene (Fragestellung 2)}

Aus den Steuerungshandlungen der Makro- und Exoebene ergeben sich für die Akteure der Meso- und Mikroebene (Grundschulleitungen und FLK) Steuerungskontexte, innerhalb derer der Einsatz der FLK innerhalb der Schulen gesteuert wird. Im Folgenden werden die Einschätzungen der Grundschulleitungen und FLK zu zwei ausgewählten Bereichen vorgestellt (Datenquelle: Projektschritt 2 und 3a, vgl. Tab. 2).

\subsubsection{Personalfragen}

Die Höhe der Ausstattung mit FLK bewerten über drei Viertel (77\%; N=78) der Grundschulleitungen und etwa $60 \%(\mathrm{~N}=47)$ der FLK als (eher) unzureichend (keine konsistenten signifikanten Unterschiede zwischen den Bundesländern). ${ }^{3}$ Die Einschätzungen der Schulleitungen und FLK spiegeln sich weitgehend in der tatsäch-

\footnotetext{
3 „Bitte bewerten Sie: Ist die Ausstattung mit förderpädagogischen Lehrkräften ausreichend, um Ihre Vor-
} stellungen von inklusivem Lernen umzusetzen?", 1 -nein, überhaupt nicht bis 5 -vollkommen ausreichend. 
lichen Ausstattung wieder, die hier anhand der Relation zwischen Gesamtschülerbzw. -schülerinnenzahl und Gesamtstunden aller FLK an der Schule (Korrelation mit der Einschätzung der Schulleitungen: 0,416, $p=0,000$; FLK: 0,345, $p=0,029$ ) sowie der Relation zwischen den Grundschullehrkräften und FLK (Korrelation mit der Einschätzung der Schulleitungen: 0,323, $p=0,004$; FLK: 0,283, $p=0,066$ ) berechnet wurde.

Die Kriterien, nach denen den Schulen Stellenanteile für FLK zugewiesen werden, halten $36 \%$ der Grundschulleitungen (27 von 75) für (eher) nicht transparent. ${ }^{4}$ Hierbei zeigen sich deutliche Unterschiede zwischen den Bundesländern: In HE wird die Transparenz im Vergleich zu NW $(p=0,008)$ und MV $(p=0,007)$ signifikant höher eingeschätzt. Etwa $65 \%$ der FLK in HE halten sie für vollkommen, weitere $12 \%$ für eher gegeben (insg. 13 von 17).

\subsubsection{Zusammenarbeit mit dem zuständigen Schulamt}

Tab. 4 zeigt Mittelwerte und Standardabweichungen zu der Zufriedenheit der Grundschulleitungen mit Zusammenarbeit mit dem zuständigen Schulamt bezüglich übergreifender Fragen zur Inklusion sowie spezifischen Fragen zu bestimmten Förderschwerpunkten. Insgesamt scheinen die Grundschulleitungen durchaus zufrieden zu sein. Die tendenziell etwas negativeren Befunde für das Bundesland HE erübrigen sich, wenn die Zufriedenheit der hessischen Schulleitungen mit der Zusammenarbeit mit den BFZs herangezogen wird, die den Grundschulen offiziell als Ansprechpartner für diese Fragen bereitstehen.

Bei übergreifenden fachlichen Fragen zur schulischen Inklusion fühlen sich vor allem die Grundschulleitungen in HE (BFZ, ca. 94\%), BE (ca. 79\%) und NW (68\%) (eher) gut unterstützt, in MV ist dieser Anteil mit $40 \%$ deutlich niedriger. Bezüglich fachlicher Fragen zu spezifischen Förderschwerpunkten scheinen die Schulämter für die Schulleitungen insgesamt ebenfalls eine gute Unterstützung zu sein: Die meisten Grundschulleitungen aus HE (BFZ, ca. 88\%) und NW (76\%) sind hiermit (eher) zufrieden, in BE (ca. 67\%) und MV (60\%) ist der Anteil etwas geringer.

Tab. 4 Zufriedenheit der Grundschulleitungen (Datenquelle: Projektschritt 2) mit der Zusammenarbeit mit dem zuständigen Schulamt (für HE zusätzlich: BFZ), 1 - überhaupt nicht zufrieden, 5 - sehr zufrieden

\begin{tabular}{|c|c|c|c|c|c|c|}
\hline \multirow[t]{2}{*}{ Item } & Gesamt & NW & $\mathrm{BE}$ & MV & $\begin{array}{l}\mathrm{HE} \\
\text { mit SSA }\end{array}$ & $\begin{array}{l}\mathrm{HE} \\
\text { mit BFZ }\end{array}$ \\
\hline & $\mathrm{M}(\mathrm{SD})$ & $\mathrm{M}(\mathrm{SD})$ & $\mathrm{M}(\mathrm{SD})$ & $\mathrm{M}(\mathrm{SD})$ & $\mathrm{M}(\mathrm{SD})$ & $\mathrm{M}(\mathrm{SD})$ \\
\hline $\begin{array}{l}\text { Inklusion, über- } \\
\text { greifend } \\
(N=75)\end{array}$ & $3,47(1,08)$ & $3,72(0,89)$ & $3,93(0,83)$ & $3,05(1,10)$ & $3,19(1,33)$ & $4,56(0,63)$ \\
\hline $\begin{array}{l}\text { Fragen zu spez. } \\
\text { FSP } \\
(N=72)\end{array}$ & $3,51(1,13)$ & $4,00(0,82)$ & $3,75(0,87)$ & $3,30(1,17)$ & $2,80(1,32)$ & $4,44(0,89)$ \\
\hline
\end{tabular}

4 „Die Kriterien, nach denen Schulen Stellenanteile für sonderpädagogische Lehrkräfte zugewiesen werden, empfinde ich als transparent. “, 1 - ̈̈berhaupt nicht bis 5 -ja. 
Innerhalb dieser Handlungskontexte stehen den Einzelschulen bzw. Grundschulleitungen verschiedene Instrumente zur Verfügung, um den Einsatz der FLK an ihrer Schule zu steuern bzw. günstige Rahmenbedingungen bereitzustellen. Dies betrifft zum einen das „Was“ der Tätigkeit der FLK, zum anderen aber auch das „Wie“ des Zustandekommens dieser Aufgaben und Aufgabenverteilungen, welches in den Abschn. 4.3 und 4.4 im Sinne eines Fokus auf die Steuerungsformen näher betrachtet wird.

\subsection{Steuerungsformen auf der Mesoebene (Fragestellung 3a)}

Welche Steuerungsformen auf der Mesoebene zu finden sind, wird anhand der Angaben der Grundschulleitungen beantwortet (Projektschritt 2, vgl. Tab. 2). Eine Möglichkeit, einen Orientierungsrahmen für die Lehrkräfte hinsichtlich des Einsatzes von FLK an der Grundschule zu schaffen, sind schulinterne schriftliche Vereinbarungen über die Aufgabenbereiche der FLK an der Schule (Arnoldt 2007, S. 129, S. $132 \mathrm{ff}$.). Insgesamt liegt eine solche Vereinbarung nach Angabe der Grundschulleitungen in weniger als der Hälfte der befragten Schulen vor (44,9\%, 35 von 78). In $\mathrm{HE}$ - wo Kooperationsverträge zwischen der Regelschule und dem abordnenden BFZ verpflichtend sind - ist dies mit $75 \%$ deutlich häufiger der Fall als in den anderen Bundesländern (MV: 21,1\%, BE: 26,7\%, NW: 53,6\%). Als Form der Vereinbarung wurden daneben vor allem Inklusionskonzepte und das Schulprogramm genannt. Kritisch zu betrachten ist die Tatsache, dass auch im Falle eines Vorliegens einer schriftlichen Vereinbarung nach Angabe der Grundschulleitungen häufig nicht alle relevanten Akteure (Grundschulleitung, die betroffene FLK, Grundschullehrkräfte) an ihrer Erstellung beteiligt waren (auf Grundlage der Projektdaten; hier nicht näher dargestellt).

Ausreichende Zeiten für Besprechungen und gemeinsame Planungen sind eine bedeutende Voraussetzung für erfolgreiche interdisziplinäre bzw. multiprofessionelle Zusammenarbeit (vgl. z. B. Grosche et al. 2020). Gerade bei nicht (schriftlich) fixierten Zuständigkeiten dürften solche Termine eine wichtige Funktion für die Auftragsklärung einnehmen. Fest und mindestens wöchentlich sind diese nach Angabe der Schulleitungen in etwa $42 \%$ (36 von 85) der Grundschulen eingerichtet. In fast ebenso vielen Schulen (insgesamt ca. 39\%, 33 von 85) gibt es solche Zeiten gar nicht bzw. nur nach Bedarf. Vor allem in MV gibt es vergleichsweise selten feste Termine (ca. 57\% gar nicht/nach Bedarf; 12 von 21). Inwieweit die FLK in die Organisation und Strukturen der Grundschule einbezogen werden, zeigt sich auch darin, inwieweit sie in die Konferenzen eingebunden werden. Tab. 5 zeigt die prozentualen Häufigkeiten der Schulen, in denen die FLK nach Angabe der Grundschulleitungen (fast) immer an den Gesamt- bzw. Lehrer- und Fachkonferenzen der Grundschule teilnehmen. In den anderen Fällen (Differenz zu 100\%) nehmen die FLK gar nicht, nur wenn Schülerinnen bzw. Schüler mit sonderpädagogischem Förderbedarf

\footnotetext{
5 „Sind an Ihrer Schule feste Besprechungs- oder Planungszeiten für Kooperationen zwischen Regelschul(und ggf. weiterem pädagogischem Personal) und sonderpädagogischen Lehrkräften vorhanden (ohne: Lehrer-/Gesamtkonferenz)?" (Nein; nach Bedarf; 1/Monat; 1/Woche; täglich).
} 
Tab. 5 Teilnahme der FLK an Konferenzen der Grundschule. (Datenquelle: Projektschritt 2, vgl. Tab. 2)

\begin{tabular}{llcccc}
\hline Konferenz & $\begin{array}{l}\text { Gesamt } \\
\text { Teilnahme (fast) immer, }\end{array}$ & $\begin{array}{c}\text { NW } \\
\text { Angabe in } \%\end{array}$ & BE & MV \\
\hline $\begin{array}{l}\text { Gesamt-/Lehrerkonferenz } \\
(N=81)\end{array}$ & 87,7 & 96,4 & 68,8 & 100 & 80,0 \\
$\begin{array}{l}\text { Fachkonferenz } \\
(N=71)\end{array}$ & 64,8 & 66,7 & 15,4 & 94,1 & 70,6 \\
\hline
\end{tabular}

betroffen sind oder nach einer anderen Regelung (z.B. nur ab einem bestimmtem Stundenkontingent an der Schule) an den Konferenzen teil.

Es zeigt sich, dass in den meisten Grundschulen die FLK (fast) immer an den Gesamt- bzw. Lehrerkonferenzen der Grundschule teilnehmen, dieser Anteil bei den Fachkonferenzen jedoch etwas niedriger ist. Auch zwischen den Bundesländern gibt es Unterschiede: So nehmen die FLK in HE deutlich seltener in den Gesamtkonferenzen, vor allem aber selten an den Fachkonferenzen teil. In BE dagegen scheint die Teilnahme der FLK an den Konferenzen der Regelfall zu sein. Als Gründe für einen seltenen bzw. eingeschränkten Einbezug der FLK werden (in Kommentarfeldern) beispielsweise genannt: Nutzung der ,Zeit für andere Aufgaben bezgl. der zu betreuenden Schüler“ (Grundschulleitung aus MV), ,zeitlich nicht machbar" (Grundschulleitung aus NW).

\subsection{Steuerungsformen auf der Mikroebene (Fragestellung 3b)}

Angesichts der bisher dargestellten Ergebnisse ist es nicht überraschend, dass die Einsatzpraktiken der FLK häufig auf Ebene einzelner bzw. kooperierender Lehrkräften ausgehandelt werden. Sie können etwa auf einer langjährigen Zusammenarbeit mit etablierten Einsatztraditionen oder auf einer als von der Grundschulleitung als ausreichend empfundenen mündlichen Auftragsklärung beruhen. Dies zeigt sich anhand einer inhaltsanalytischen Auswertung der Interviews mit 37 FLK (Datenquelle: Projektschritt 3b, vgl. Tab. 2), in der vier vorrangige Verabredungsmodi identifiziert werden konnten (vgl. Abb. 2): Am Häufigsten $(n=18)$ wurde eine kollegiale Aushandlung mit den anderen Lehrkräften der Grundschule genannt. In 8 Fällen beruhten die Einsatzpraktiken auf eigenen Entscheidungen der befragten FLK und in 6 Fällen wurden die individuellen Aufgabenbereiche gemeinsam mit der Grundschulleitung entwickelt. In weiteren fünf Fällen wurden die Aufgaben und Aufgabenverteilungen aus bereits bestehenden Traditionen der Schule oder Regelungen mit anderen (bereits oder zuvor an der Schule tätigen) FLK übernommen.

Zwischen den Bundesländern zeigen sich tendenzielle Unterschiede, die jedoch mit Blick auf die geringe Stichprobengröße zurückhaltend interpretiert werden müssen: In HE, wo schriftliche Vereinbarungen zum Einsatz der FLK verpflichtend sind (s. Abschn. 4.3), werden die individuellen Aufgabenbereiche der FLK anteilig seltener auf Ebene der kooperierenden Lehrkräfte ausgehandelt (ca. 27\%). Hier werden sie im Gegensatz zu den anderen Bundesländern häufiger mit den Schulleitungen (ggf. im Rahmen der Erstellung der Kooperationsvereinbarung) entwickelt (ca. 36\%). In MV und BE scheint eine Aushandlung zwischen den Lehrkräften dagegen der Regelfall zu sein (80\% und ca. 64\%). Im Bundesland BE wurde dies et- 
Abb. 2 Vorrangige Verabredungsmodi der förderpädagogischen Lehrkräfte (FLK) über Aufgaben und Aufgabenverteilungen; Ergebnisse auf Basis der FoLiS-Studie. (Lesehinweis (Beispiel): NW 2/9=zwei von neun FLK in NW)

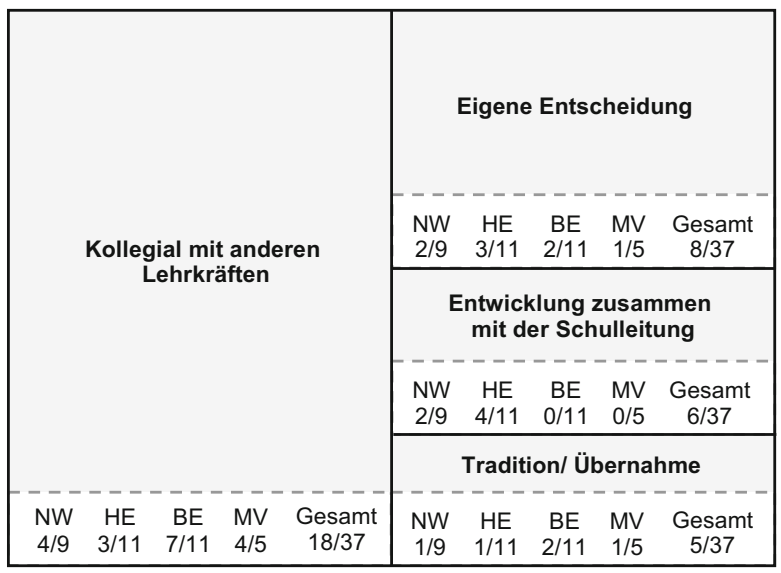

wa damit begründet, dass es schulinterne „Fachkonferenzen Sonderpädagogik“ (2x) bzw. Klassenteamstunden $(2 \times)$ gibt.

Zufrieden mit der Art des Verabredungsmodus waren vor allem die FLK, die ihre Aufgabengebiete kollegial mit den anderen Lehrkräften der Schule verabredet haben (12 von 18). Unter den FLK des Verabredungsmodus Entwicklung zusammen mit der Schulleitung zeigen sich ebenfalls zwei Drittel (4 von 6) zufrieden, u.a. aufgrund der großen Bereitschaft der Grundschulleitung zu Anpassungen der Aufgabengebiete an die Bedürfnisse der Schülerinnen und Schüler. Im Modus Eigene Entscheidung waren drei von acht FLK zufrieden, u.a. da die Schulgemeinschaft ihnen die Kompetenz über das „Wie“ der Förderung vollständig zuspricht und ihnen damit zugleich die Ausgestaltung des eigenen Einsatzes überlässt.

\section{Zusammenfassung und Diskussion}

Der Artikel untersuchte die Steuerungsformen der Akteure im Mehrebenensystem Schule in Bezug auf den Einsatz von FLK an Grundschulen. Inspiriert durch den Forschungsansatz der EG, der insbesondere die Regelungsstrukturen in Bildungssystemen analysiert, wurden in vier Bundesländern Vertreter und Vertreterinnen der Systemebenen hinsichtlich ihrer Steuerungsaktivitäten befragt und in ihrer jeweiligen Kopplung betrachtet. Tab. 6 zeigt - als Extrakt der empirischen Untersuchung die jeweiligen Steuerungskontexte, -formen und -ergebnisse in Bezug auf den Einsatz von FLK an Grundschulen.

Es konnte festgestellt werden, dass bezüglich des Einsatzes von FLK an Grundschulen auf der Makro- und Exoebene eine traditionelle inputorientierte Steuerung vorliegt, die sich vor allem auf die Bereitstellung von Ressourcen und Organisationsmodellen konzentriert. Definitionen von Outputs schulischer Inklusion finden sich nicht. Die konkrete Ausformung schulischer Inklusion bezüglich des Einsatzes der FLK wird mit Verweis auf Schulautonomie und (angenommener) Schulleitungskompetenz delegiert: „Sie wissen ja, welche Schätze sie im Kollegium haben“ 
Tab. 6 Steuerungskontexte, -formen und -ergebnisse bezüglich des Einsatzes von FLK an inklusiven Grundschulen im Mehrebenenmodell. (Ergebnisse auf Grundlage der FoLiS-Studie)

\begin{tabular}{|c|c|c|}
\hline Ebene & Steuerungskontext & Steuerungsformen und -ergebnisse \\
\hline $\begin{array}{l}\text { Makroebene } \\
\text { (Bildungs- } \\
\text { politik) }\end{array}$ & $\begin{array}{l}\text { UN-BRK, Inklusion als gesellschaftli- } \\
\text { che Forderung }\end{array}$ & $\begin{array}{l}\text { Ratifizierung der und Anpassung gesetzlicher } \\
\text { Regelungen an die UN-BRK }\end{array}$ \\
\hline $\begin{array}{l}\text { Exoebene } \\
\text { (Bildungs- } \\
\text { verwaltung) }\end{array}$ & $\begin{array}{l}\text { Umsetzung der gesetzlichen und } \\
\text { untergesetzlichen Vorgaben, landes- } \\
\text { spezifische Aktionspläne/Leitfäden, } \\
\text { Inklusion als Aufgabengebiet der } \\
\text { Schulentwicklung, Aufgabe der Perso- } \\
\text { nalzuweisung }\end{array}$ & $\begin{array}{l}\text { Aufgabenwahrnehmung gemäß Landesrecht, } \\
\text { ggf. Einrichtung weiterer Akteure, Umsetzung } \\
\text { bildungspolitischer Entscheidungen } \\
\text { - Bedarfserhebung } \\
\text { - Stellenberechnung } \\
\text { - Personalrekrutierung } \\
\text { - Koordination Schule \& FLK }\end{array}$ \\
\hline $\begin{array}{l}\text { Mesoebene } \\
\text { (Einzelschule) }\end{array}$ & $\begin{array}{l}\text { Einsatz gemäß Organisationsmodell } \\
\text { bei gegebener pädagogischen Schul- } \\
\text { autonomie }\end{array}$ & $\begin{array}{l}\text { Steuerung oder Delegierung des Einsatzes } \\
\text { der FLK, schulinterne Regelungen (z.B. } \\
\text { Besprechungen), Einbindung der FLK in } \\
\text { schulinterne Gremien }\end{array}$ \\
\hline $\begin{array}{l}\text { Mikroebene } \\
\text { (Lehrkräfte) }\end{array}$ & $\begin{array}{l}\text { Beachtung der rechtlichen Vorgaben } \\
\text { und Aufgabenbeschreibung, inner- } \\
\text { schulische Vereinbarungen }\end{array}$ & $\begin{array}{l}\text { Organisation der Unterrichtsverpflichtung } \\
\text { (z.B. Doppelbesetzungen, Kleingruppenför- } \\
\text { derung, eigener Unterricht) und weiterer Auf- } \\
\text { gaben in Absprache mit anderen Lehrkräften }\end{array}$ \\
\hline
\end{tabular}

(Interview L1_C_T4). Begründet wird dies mit der Notwendigkeit eines variablen und schulspezifischen Einsatzes auf Ebene der Einzelschule. Gleichzeitig werden den Akteuren dieser Ebenen weder konkrete Zielformulierungen noch entsprechende Instrumente der Evaluation ihrer schulspezifischen Umsetzung inklusiver Bildung (Mesoebene, Einzelschule) bzw. ihrer sonderpädagogischen Tätigkeit (Mikroebene, Lehrkräfte) - als Bestandteil und Kennzeichen einer outputorientierten Steuerung bereitgestellt. Diese fragmentarische Steuerung führt zu einer großen Verantwortung der Einzelschule (und der FLK selber) in der Ausgestaltung des Einsatzes der FLK, wobei sich die Grundschulleitungen mit der fachlichen Unterstützung durch die Schulämter bzw. BFZs insgesamt zufrieden zeigen. Auf Ebene der Einzelschulen zeigt sich, dass die FLK nicht in allen Bundesländern gleich regelmäßig an den Konferenzen der Grundschule teilnehmen, Besprechungs- und Planungszeiten nicht ausreichend und flächendeckend implementiert sind und schriftliche Vereinbarung zur Klärung der Aufgabenbereiche der FLK selten und aufgrund des fehlenden Einbezugs aller relevanten Akteure nicht in ihrem vollen Potenzial genutzt werden. Von den FLK erfordert diese geringe vorausgehende Strukturgebung die Fähigkeit zu einer individuellen Rollenfindung, was auch zukünftig als Herausforderung der Professionalisierung angenommen werden kann.

Limitationen des Beitrags und der dargestellten Ergebnisse ergeben sich durch die geringe Stichprobengröße und fehlende Angaben einiger Schulleitungen und FLK in den Fragebögen. Des Weiteren wurden lediglich vier der 16 deutschen Bundesländer sowie eine nicht-repräsentative Auswahl an Schulamtsbereichen, Grundschulen und FLK beforscht. Es wurde nicht systematisch erhoben, welche eigenen Definitionen und Outputs (i. S. von Qualität schulischer Inklusion) die Akteure entwickeln und ob ihre Handlungs-/Steuerungsformen diese Ziele kohärent verfolgen (für die innerschulische Entwicklung s. Bengel 2021; für die Akteure Schulaufsicht 
und Schulleitung s. Klein und Bremm 2020). Sofern in Folgeuntersuchungen solche Definitionen und Outputs gefunden werden können, wäre eine Kongruenz oder Nicht-Kongruenz innerhalb und zwischen den Ebenen zu untersuchen. In weiteren Studien sollte auch die Perspektive anderer beteiligter Professionen, insbesondere der Grund- bzw. Regelschullehrkräfte, sowie die Verortung der neuen Akteure (s. Abschn. 4.1) im Mehrebenensystem Schule und deren Steuerungsaktivitäten einbezogen werden. Auch inwieweit die allmähliche Veröffentlichung von Aufgabenbeschreibungen durch die Exoebene zu einer Veränderung des Einsatzes der FLK führt, ist in Folgeuntersuchungen zu erheben.

Zusammenfassend zeigen sich Hinweise darauf, dass hinsichtlich des Einsatzes der FLK an Grundschulen auf der Makro- und Exoebene eine fragmentarische Steuerung vorliegt, die im Widerspruch mit der outputorientierten und an Selektion festhaltenden Steuerung des Schulwesens steht (,Wie soll Inklusion möglich sein in einem System, das strukturell auf Selektion angelegt ist?“ Oelkers 2012, S. 34), mit bekannten Herausforderungen der Mesoebene (z. B. Lehrkräftemangel, nicht inklusive Schulkulturen) kollidiert und die Verantwortung für die Ausgestaltung inklusiver Bildung in weiten Teilen den Akteuren der Meso- und Mikroebene überlässt.

Funding Open Access funding enabled and organized by Projekt DEAL.

Open Access Dieser Artikel wird unter der Creative Commons Namensnennung 4.0 International Lizenz veröffentlicht, welche die Nutzung, Vervielfältigung, Bearbeitung, Verbreitung und Wiedergabe in jeglichem Medium und Format erlaubt, sofern Sie den/die ursprünglichen Autor(en) und die Quelle ordnungsgemäß nennen, einen Link zur Creative Commons Lizenz beifügen und angeben, ob Änderungen vorgenommen wurden.

Die in diesem Artikel enthaltenen Bilder und sonstiges Drittmaterial unterliegen ebenfalls der genannten Creative Commons Lizenz, sofern sich aus der Abbildungslegende nichts anderes ergibt. Sofern das betreffende Material nicht unter der genannten Creative Commons Lizenz steht und die betreffende Handlung nicht nach gesetzlichen Vorschriften erlaubt ist, ist für die oben aufgeführten Weiterverwendungen des Materials die Einwilligung des jeweiligen Rechteinhabers einzuholen.

Weitere Details zur Lizenz entnehmen Sie bitte der Lizenzinformation auf http://creativecommons.org/ licenses/by/4.0/deed.de.

\section{Literatur}

Altrichter, H., \& Maag-Merki, K. (2016). Steuerung der Entwicklung des Schulwesens. In H. Altrichter \& K. Maag-Merki (Hrsg.), Handbuch Neue Steuerung im Schulsystem. Wiesbaden: Springer VS. https:// doi.org/10.1007/978-3-531-18942-0_1.

Arnoldt, B. (2007). Kooperationsformen - Bedingungen für gelingende Zusammenarbeit. In H.-G. Holtappels, E. Klieme, T. Rauschenbach \& L. Stecher (Hrsg.), Ganztagsschule in Deutschland. Ergebnisse der Ausgangserhebung der „Studie zur Entwicklung von Ganztagsschulen“ (StEG) (S. 123-136). Weinheim: Juventa.

Bengel, A. (2021). Schulentwicklung Inklusion. Empirische Einzelfallstudie eines Schulentwicklungsprozesses. Bad Heilbrunn: Klinkhardt.

Berkemeyer, N. (2010). Die Steuerung des Schulsystems. Theoretische und praktische Explikationen. Wiesbaden: Springer.

Bezirksregierung Düsseldorf (2020). Gemeinsames Lernen auf dem Weg zur Inklusion. Manual zur Erstellung eines schulischen Konzepts in der allgemeinen Schule (Mai 2020). https://www.brd.nrw. de/system/files/migrated_documents/Inklusion-Manual_Gemeinsames_Lernen_60a3fa0d13207.pdf. Zugegriffen: 12. Sept. 2021. 
Bezirksregierung Köln (2019). Inklusion an Schulen im Regierungsbezirk Köln. https://www.bezregkoeln.nrw.de/brk_internet/leistungen/abteilung04/generalien/inklusion/inklusion.pdf. Zugegriffen: 12. Sept. 2021.

Budde, J. (2018). Erziehungswissenschaftliche Perspektiven auf Inklusion und Intersektionalität. In T. Sturm \& M. Wagner-Willi (Hrsg.), Handbuch schulische Inklusion (S. 45-60). Opladen: Budrich.

Fend, H. (2008). Schule gestalten. Systemsteuerung, Schulentwicklung und Unterrichtsqualität. Wiesbaden: Springer VS.

Fuchs, H.-W. (2008). Educational Governance und Neue Steuerung: Grundsätze - Beispiele - Erwartungen. In R. Langer (Hrsg.), Warum tun die das? Governanceanalysen zum Steuerungshandeln in der Schulentwicklung (S. 19-30). Wiesbaden: Springer VS.

Gasterstädt, J. (2019). Der Komplexität begegnen und Inklusion steuern. Eine Situationsanalyse zur Umsetzung von Artikel 24 der UN-BRK in Deutschland. Wiesbaden: Springer VS.

Grosche, M., Fussangel, K., \& Gräsel, C. (2020). Kokonstruktive Kooperation zwischen Lehrkräften. Aktualisierung und Erweiterung der Kokonstruktionstheorie sowie deren Anwendung am Beispiel schulischer Inklusion. Zeitschrift für Pädagogik, 66(4), 461-479.

Grummt, M. (2019). Sonderpädagogische Professionalität und Inklusion. Wiesbaden: Springer VS.

Heinrich, M., Urban, M., \& Werning, R. (2013). Grundlagen, Handlungsstrategien und Forschungsperspektiven für die Ausbildung und Professionalisierung von Fachkräften für inklusive Schule. In H. Döbert \& H. Weishaupt (Hrsg.), Inklusive Bildung professionell gestalten (S. 69-133). Münster: Waxmann.

HKM (Hessisches Kultusministerium) (2020). Das inklusive Schulbündnis (iSB). Leitfaden zu den Aufgaben und der Organisation der inklusiven Schulbündnisse. https://kultusministerium.hessen.de/ sites/default/files/media/hkm/inklusive_schulbuendnisse_isb_-_leitfaden_zu_den_aufgaben_und_ der_organisation_der_inklusiven_schulbuendnisse_0.pdf. Zugegriffen: 12. Sept. 2021.

Hochschulrektorenkonferenz und Kultusministerkonferenz (2015). Lehrerbildung für eine Schule der Vielfalt. Gemeinsame Empfehlung von Hochschulrektorenkonferenz und Kultusministerkonferenz. Beschluss der KMK vom 12.03.2015/Beschluss der HRK vom 18.03.2015

Holder, K., \& Kessels, U. (2018). Lehrkräfte zwischen Bildungsstandards und Inklusion: Eine experimentelle Studie zum Einfluss von „Standardisierung“ und „Individualisierung“ auf die Bezugsnormorientierung. Unterrichtswiss 46, 87-104. https://doi.org/10.1007/s42010-018-0013-0

Klein, E. D., \& Bremm, N. (2020). Unterstützung - Kooperation - Kontrolle. Zum Verhältnis von Schulaufsicht und Schulleitung in der Schulentwicklung. Wiesbaden: Springer VS.

KMK (2011). Inklusive Bildung von Kindern und Jugendlichen mit Behinderungen in Schulen. Beschluss der Kultusministerkonferenz vom 20.10.2011

KMK (2014). Standards für die Lehrerbildung: Bildungswissenschaften. Beschluss der Kultusministerkonferenz vom 16.12.2004 i.d. F. vom 16.05.2019

KMK For.mat Perspektivenwechsel im Bildungssystem: Vom input- zum outcomeorientierten Denken. http://www.kmk-format.de/material/Fremdsprachen/1-2-7_Outcome-Orientierung.pdf. Zugegriffen: 12. Sept. 2021.

Kuhl, J., Moser, V., Dietze, T. \& Wolf, L. (2020): Schlussbericht des BMBF-Forschungsprojektes Förderpädagogische Lehrkräfte in inklusiven Schulen (FoLis).

Kussau, J., \& Brüsemeister, T. (2007). Educational Governance: Zur Analyse der Handlungskoordination im Mehrebenensystem der Schule. In H. Altrichter, T. Brüsemeister \& J. Wissinger (Hrsg.), Handlungskoordination und Steuerung im Bildungssystem (S. 15-54). Wiesbaden: Springer VS.

LRH NI (Niedersächsischer Landesrechnungshof) (2018). Jahresbericht 2018 des Niedersächsischen Landesrechnungshofs. https://www.lrh.niedersachsen.de/download/131387/Jahresbericht_2018.pdf. Zugegriffen: 12. Sept. 2021.

Mayntz, R. (1997). Politische Steuerung und gesellschaftliche Steuerungsprobleme - Anmerkungen zu einem theoretischen Paradigma. In R. Mayntz (Hrsg.), Soziale Dynamik und politische Steuerung (S. 186-208). Frankfurt a. M.: Campus.

MSB NRW (Ministerium für Schule und Bildung des Landes NRW) (2019). Das Schulwesen in Nordrhein-Westfalen aus quantitativer Sicht 2018/19. Statistische Übersicht 404. Düsseldorf: MSB NRW (Ministerium für Schule und Bildung des Landes NRW).

MSN NRW (Ministerium für Schule und Weiterbildung des Landes NRW) (2009). Das Schulwesen in Nordrhein-Westfalen aus quantitativer Sicht 2008/09. Statistische Übersicht 369. Düsseldorf: Ministerium für Schule und Weiterbildung des Landes NRW.

Neumann, P. (2019). Kooperation selbst bestimmt? Interdisziplinäre Kooperation und Zielkonflikte in inklusiven Grundschulen und Förderschulen. Münster: Waxmann. 
Oelkers, J. (2012). Inklusion als Aufgabe der öffentlichen Schule. In S. Seitz, N.-K. Finnern, N. Korff \& K. Scheidt (Hrsg.), Inklusiv gleich gerecht? (S. 32-45). Bad Heilbrunn: Klinkhardt.

Piezunka, A. (2020). Ist eine gute Schule eine inklusive Schule? Entwicklung von Messinstrumenten durch Schulinspektionen. Bad Heilbrunn: Klinkhardt.

Rürup, M. (2015). Educational Governance. In I. Hedderich, G. Biewer, J. Hollenweger \& R. Markowetz (Hrsg.), Handbuch Inklusion und Sonderpädagogik (S. 684-689). Bad Heilbrunn: Klinkhardt.

Rürup, M. (2020). Schulautonomie im Ländervergleich. Eigenständigkeit von Einzelschulen als politisches Programm. Pädagogik, 71(6), 44-47.

Tegge, D. (2020). Inklusion als schulischer Transformationsprozess. Möglichkeiten und Grenzen der indikatorengestützten Darstellung des Gemeinsamen Lernens auf kommunaler und Einzelschulebene. Bad Heilbrunn: Klinkhardt.

Zlatkin-Troitschanskaia, O. (2006). Steuerbarkeit von Bildungssystemen mittels politischer Reformstrategien - Interdisziplinäre theoretische Analyse und empirische Studie zur Erweiterung der Autonomie im öffentlichen Schulwesen. Frankfurt am Main: Peter Lang. 